\title{
PROPORTIONALLY FAIRER PUBLIC SERVICE SYSTEMS DESIGN
}

This paper focuses on the utilitarian solution of public service system design problem, obtained when maximising the sum of all utilities and proportionally fair-like solution, taking into account proportional changes in individual utilities. As an archetypal example of the optimisation problem, we are examining the weighted p-median problem, which is solved by the primary-dual based procedure. We use realistic largescale data describing the road network and spatial distribution of population. By comparing the resulting solutions, for selected range of parameters, we evaluate how costly it is to consider fairness criteria in the service system design. As for integer problems the proportional fairness scheme does not guarantee the existence of dominant optimal solution, we evaluate the close neighbourhood of obtained solutions. Based on these analyses we draw conclusions on the price of (proportionally-like) fair solutions and their stability.

Keywords: Facility location, system optimum, proportional fairness, price of fairness.

\section{Introduction}

Designing public service systems, as for example, distribution systems, emergency medical systems, or to decide on positions of marshalling yards throughout the railway network, various types of location and allocation models can be used [1]. In general, this problem can be seen as an example of resource allocation problem, with a central planner. Although all serviced customers typically share the costs for system construction and its maintenance, not all customers (citizens) are enjoying the same access to services. Therefore the question to what extent is the resulting system design fair is highly relevant. If the satisfaction level of customers with a given situation can be estimated by a utility function, various fairness schemes can be used to compare corresponding optimal allocation strategies.

Typically these schemes are either aiming at the systems efficiency or they are trying to achieve a certain level of fairness. Eventually, it is required to find a reasonable trade-off between them. One of the most frequently discussed decision schemes, focusing on the system efficiency, is the utilitarian solution obtained when maximising the sum of all utilities. Such scheme does not include any individual notion of fairness. Commonly used fairness schemes are the max-min fair (MMF) scheme, sequentially maximising the utilities of those who are the least well off and the proportionally fair (PF) distribution compromising between the efficiency and the fairness. When following the latter scheme, a transfer of resources between two customers is favourable when the relative increase in the utility of one customer is larger than the corresponding aggregated relative decrease in the utility of all other customers. Fairness schemes have been thoroughly studied in many areas. For example, MMF was discussed in the context of the set partitioning problem [2] or flow problems [3]. PF was mainly used as a mechanism for routing flows in communication networks [4].

Recently, simple measure, the price of fairness [5], has been proposed and analytically studied on problems with compact and convex utility set. Adopting this concept, the overall objective of this paper is to numerically study the loss of the system efficiency, when calculating close to PF solutions of the weighted p-median problem.

\section{Problem description}

The weighted p-median problem [6] is one of the most basic and important models for solving the problems related to the public service design [1]. The locations of at maximum $p_{\max }$ servers servicing the set of customers $J$ are to be selected from the set of candidate locations $I$. Each customer $j \in J$ is serviced by one server $i \in I$. The decision on assigning the customer $j$ to the server location $i$ is represented by the binary variable $z_{i j}$. The corresponding utility is a function of the travelling distance $d_{i j}$ between the server site $i$ and the customer's home $j$. Hereafter, we stipulate the utility of the customer $j$ with respect to the server location $i$ to be $u_{i j}=$ $=d_{\text {max }}^{j}+1-d_{i j}$, where $d_{\text {max }}^{j}=\max \left[d_{i j}: i \in I\right]^{1)}$. This allows

\footnotetext{
* Lubos Buzna, Michal Kohani, Jaroslav Janacek

Department of Transportation Networks, Faculty of Management Science and Informatics, University of Zilina, Slovakia, E-mail: lubos.buzna@fri.uniza.sk

1) This choice of the utility function was mainly motivated by preserving the type of the relation between $d_{i j}$ and $u_{i j}$ as it is perceived by customers (the closer is the server the larger is the utility of the customer). Alternatively, as $d_{\max }^{j}+1$ value can be chosen sufficiently large positive number. However, as the large values can be problematic when evaluating the results, we use the smallest possible values which make sure that $u_{i j}>0$
} 
us to obtain the utilitarian solution $Z_{U}$ of the weighted $p$-median problem when optimising the following objective function:

$$
\operatorname{SYSTEM}(Z)=\sum_{i \in I} \sum_{j \in J} b_{j} u_{i j} z_{i j}
$$

where $b_{j}$ is the number of customers occupying location $j$.

For optimisation problems with convex and compact utility set, the PF solution exists and can be found by maximising the product of individual utilities of customers over set $J$ [4]. Under these assumptions it is equivalent to the maximising the sum of logarithms of all utilities. Unfortunately, the weighted $p$-median problem is discrete and thus the utility set does not fulfil these conditions. Therefore the optimal solution $Z_{P}$ obtained by optimising the objective function:

$$
P(Z)=\sum_{j \in J} b_{j} \log \left(\sum_{i \in I} u_{i j} z_{i j}\right)=\sum_{j \in J} \sum_{i \in I} b_{j} \log \left(u_{i j}\right) z_{i j}
$$

should be seen as an approximate solution, which provides proportionally fairer (but not necessarily the most fair) solution than utilitarian solution ${ }^{2)}$. Please note, that this formulation assumes for every $j$ only one $z_{i j}$ variable taking value of 1 . In order to compare these two solutions, following reference [5], the price of proportionally fairer solution can be defined as:

$$
\operatorname{PoF}=\frac{\operatorname{SYSTEM}\left(Z_{U}\right)-\operatorname{SYSTEM}\left(Z_{P}\right)}{\operatorname{SYSTEM}\left(Z_{U}\right)}
$$

\section{Model formulation}

To formulate the utility allocation problem, we make use of the above introduced notation, where $J$ represents the set of serviced customers and $I$ is the set of possible server locations.

The objective is to determine at most $p_{\max }$ locations from the set $I$ so that the sum of utilities (1) and the sum proportional utilities (2) perceived by each customer from the set $J$ are maximum. The location-allocation model of the problem can be can be formulated by introducing following decision variables.

Variables $y_{i} \in\{0,1\}$ model a decision on server location at place $i \in I$. The variable $y_{i}$ takes the value of 1 if a server is located at $i$ and it takes the value of 0 otherwise. In addition, the allocation variables $z_{i j} \in\{0,1\}$ for each $i \in I$ and $j \in J$ are introduced to assign customer $j$ to possible server location $i\left(z_{i j}=1\right)$. Then the location-allocation model follows.

Maximise (1) or (2)

$$
\begin{array}{ll}
\text { Subject to } & \sum_{i \in I} z_{i j}=1 \quad \text { for } j \in J \\
& z_{i j} \leq y_{i} \quad \text { for } i \in I \text { and } j \in J \\
& \sum_{i \in I} y_{i} \leq p_{\max } \\
z_{i j} \in\{0,1\} \quad \text { for } i \in I \text { and } j \in J \\
y_{i} \in\{0,1\} \quad \text { for } i \in I .
\end{array}
$$

In the above model, the allocation constraints (4) ensure that each customer is assigned to exactly one possible server location. Link-up constraints (5) enable to assign a customer to a possible server location $i$ only if a server is located at this location and constraint (6) limits the number of located servers.

Both models (1), (4)-(8) and (2), (4)-(8) can be easily reformulated as the classical weighted $p$-median problem by using the following rearrangement. We make use of the fact that both sets $J$ and $I$ are finite and we can define $U^{\max }$ as $\max \left\{u_{i j}: i \in I, j \in J\right]$. Denoting $q_{i j}-U^{\max }-u_{i j}$ as a nonnegative distance from $j$ to $i$ and considering constraints (4) we can derive the relation:

$$
\begin{aligned}
& \sum_{i \in I} \sum_{j \in J} b_{j} u_{i j} z_{i j}=U^{\max } \sum_{j \in J} b_{j}-U^{\max } \sum_{j \in J} b_{j}+\sum_{i \in I} \sum_{j \in J} b_{j} u_{i j} z_{i j}= \\
& =U^{\max } \sum_{j \in J} b_{j}-\left(U^{\max } \sum_{j \in J} b_{j} \sum_{i \in I} z_{i j}-\sum_{i \in I} \sum_{j \in J} b_{j} u_{i j} z_{i j}\right)= \\
& =U^{\max } \sum_{j \in J} b_{j}-\left(\sum_{i \in I} \sum_{j \in J} b_{j}\left(U^{\max }-u_{i j}\right) z_{i j}\right)= \\
& =U^{\max } \sum_{j \in J} b_{j}-\sum_{i \in I} \sum_{j \in J} b_{j} q_{i j} z_{i j}
\end{aligned}
$$

As the first item of the resulting expression is a constant, we can obtain an optimal solution of (1), (4)-(8) by maximising the second term only. The maximisation can be replaced by minimisation by multiplying objective function by -1 and thus instead of maximising (1) subject to (4)-(8) we can minimise (9) subject (4)-(8), which is the classical weighted p-median problem.

$$
\text { Minimize } \sum_{i \in I} \sum_{j \in J} b_{j} q_{i j} z_{i j} \text {, }
$$

The same adjustment can be done with the proportional utility (2) by replacing $u_{i j}$ with $\log \left(u_{i j}\right)$.

\section{Numerical experiments}

Taking an initial step in investigating the $P o F$ for the public service system design problems, we use three datasets describing the road network and spatial distribution of population for three selected large geographical areas (see Fig. 1). It is assumed that all inhabitants are customers of the public service system. For each

\footnotetext{
2) As for discrete utility sets the transitivity property of dominance between various solutions does not have to hold, the existence of the most proportionally fair solution is not guaranteed.
} 
area we created two subsets of problems characterised by the size of the set $I$ (for more detail see caption of Fig. 2).

In Fig. 2 we varied the parameter $p_{\max }$ and calculated the weighted $p$-median problem considering objective functions given by Eq. (1) and Eq. (2), respectively. The weighted $p$-median problem is solved by combining the Lagrangian relaxation of the constraint limiting the number of located servers to $p_{\max }$ with the primal-dual procedure solving the uncapacitated facility location problem [7]. As the obtained results show, when evaluating both solutions by using Eq. (3), surprisingly, we are getting very small difference between them, finding $P o F$ value close to value zero. The only one significant exception are cases when $p_{\max }$ takes low values. Here we are locating small number of servers and $P o F$ is more sensitive to the particular choice of sites. However, even here $P o F$ exceeded value 0.01 only in few cases.

As mentioned earlier, for discrete optimisation problems, optimising the objective function given by Eq. (2) does not have to necessarily result in finding the most proportionally fair solution. Thus, not only for the utilitarian solution given by Eq. (1) but also for the solution obtained by Eq. (2), we may expect that a replace-

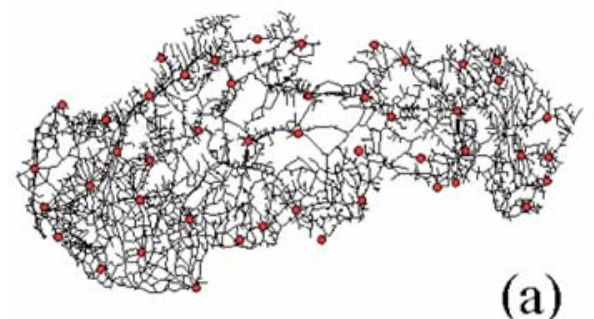

(a)

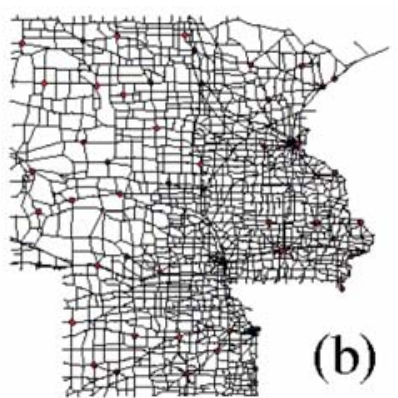

Fig. 1 Examples of used datasets: (a) road network of Slovak Republic with $|I|=47$; (b) joined road networks of six northern US states [7] (Iowa, Kansas, Minnesota, Nebraska, North Dakota and South Dakota) with $|I|=50 ;$ (c) joined road networks of six southern US states [8] (Alabama, Georgia, Mississippi, North Carolina, South Carolina) with $|I|=51$
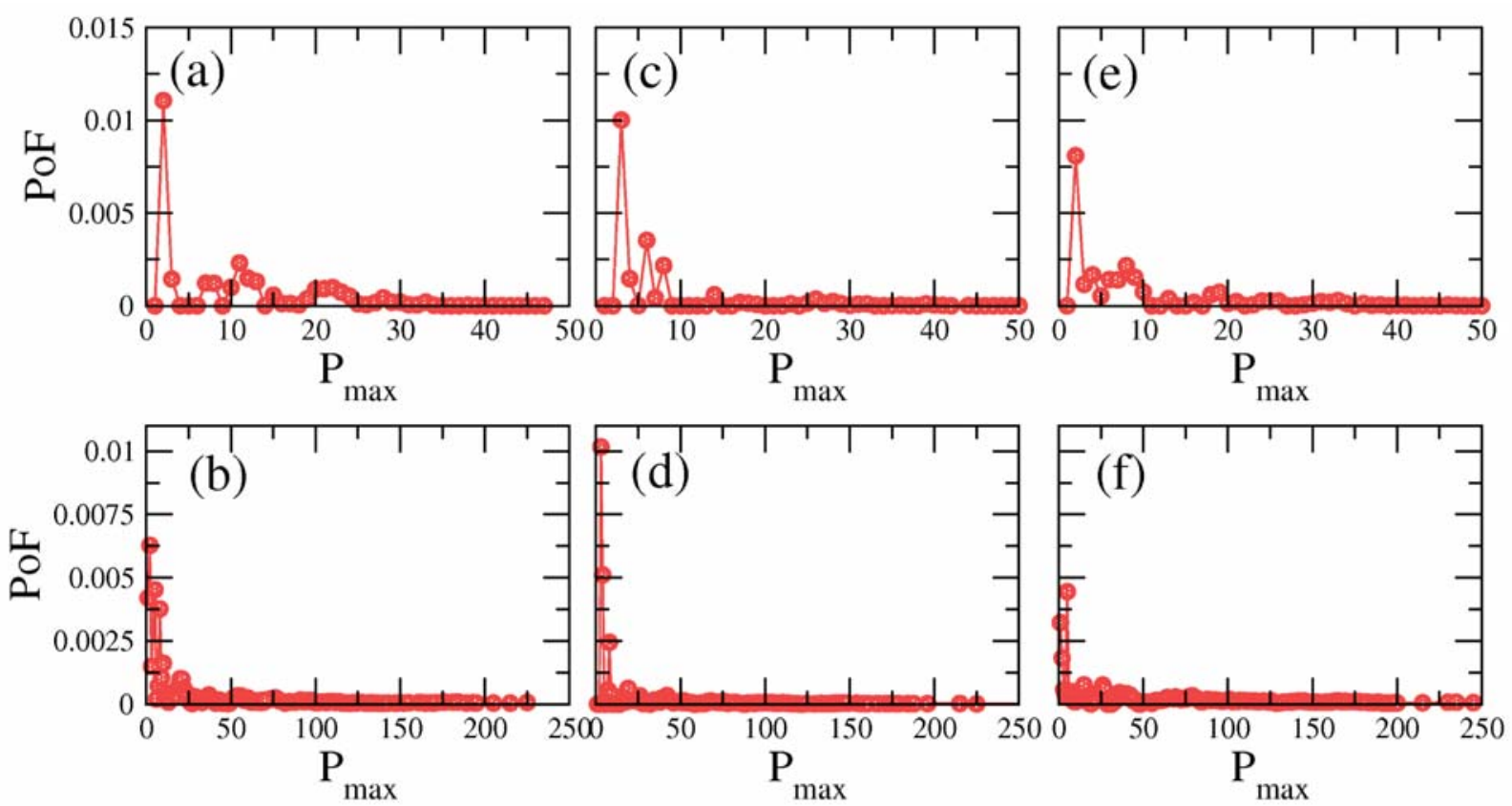

Fig. 2 Values of the price of fairness (PoF) obtained by evaluating Eq.(3) for solutions obtained by considering criteria SYSTEM(ZU) and SYSTEM(ZP). (a) and (b) correspond to experiments with road network of Slovak Republic with $|I|=47$ and $|I|=516$, respectively; (c) and (d) correspond to experiments with joined road networks of six northern US states with $|I|=50$ and $|I|=500$, respectively; and (e) and ( $f$ ) correspond to experiments with joined road networks of six southern US states with $|I|=51$ and $|I|=499$, respectively 

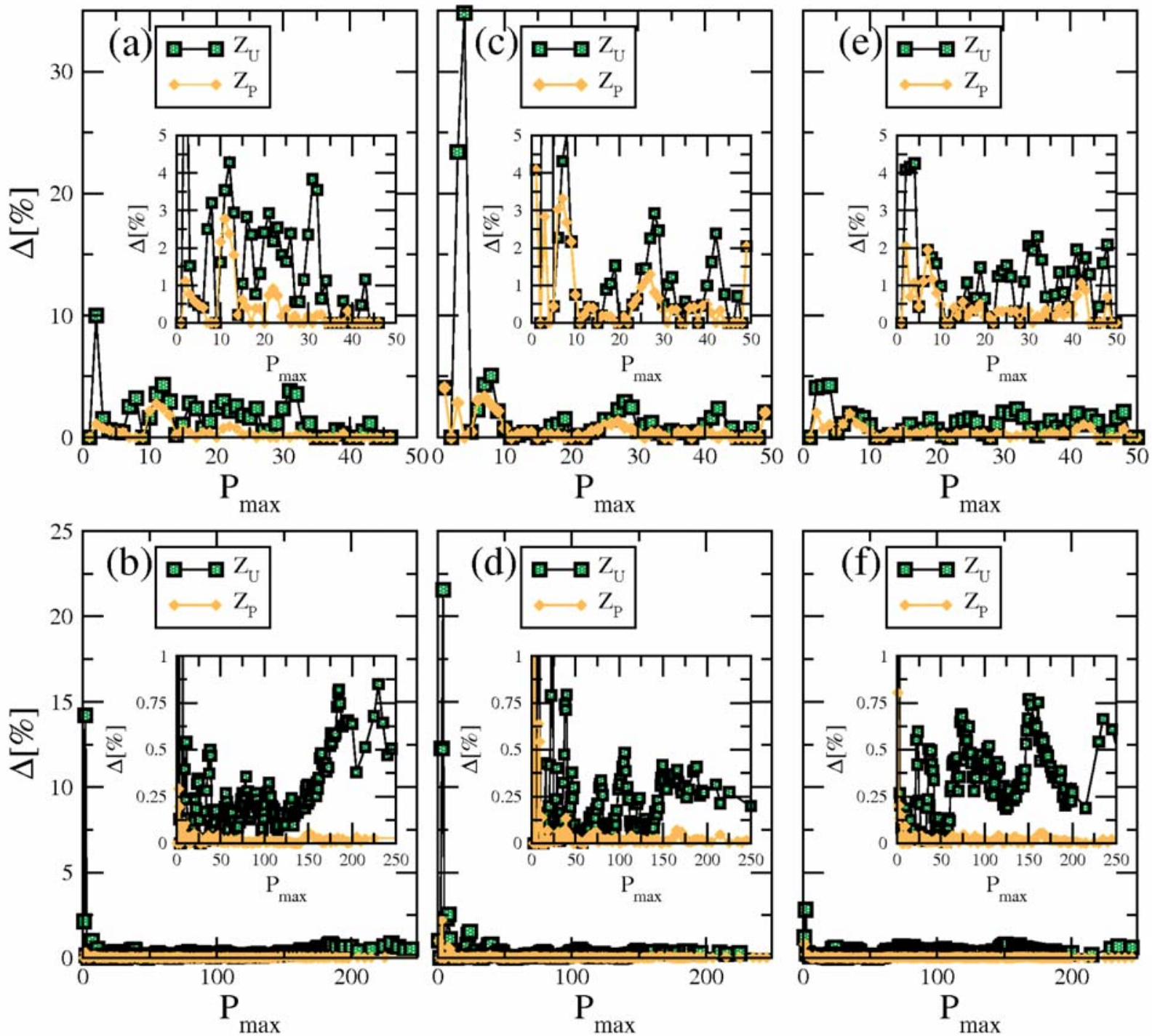

Fig. 3 Fraction of proportionally fairer neighbouring solutions obtained by swapping one occupied for one unoccupied server site. To improve the readability of figures the inset shows a zoom of the region with tiny $\Delta$ values. The labels correspond to experiments on different datasets organised similarly as in Fig. 2

ment of some located servers can result in (proportionally) fairer situation. In order to quantify such tendency we evaluate the closest neighbourhood of reached solutions. This neighbourhood is defined as the set of all solutions, which can be obtained by swapping one occupied server location for one empty server location. In Fig. 3 is evaluated the percentage of solutions which are proportionally fairer than optimal solutions $Z_{U}$ and $Z_{P}$. The obtained results show that solutions $Z_{P}$ have clearly smaller number of proportionally fairer solutions in the close neighbourhood than solutions $Z_{U}$. Thus, the results also confirm that from the point of view of PF scheme it is sensible to apply objective function (2) to the weighted p-median problem.

\section{Conclusions}

Applying PF criterion, known from the utility theory, and comparing it with utilitarian solution we evaluated numerically the price of fairness for the weighted p-median problem. The obtained results show:

- locally increased level of fairness when using PF criterion,

- and, surprisingly small price of fairness, although the number of customers is very large.

These two main findings suggest that for real-world problems, where the weighted $p$-median problem could be a relevant modelling tool, it can be relatively easy to improve the fairness of the 
system design as the (proportionally-like) fair allocation is close to the efficient allocation. However, to be able to present practically more relevant conclusions further steps are needed in several areas and we need:

- to construct and to investigate more natural utility functions, (this will probably require to reformulate the problem considering negative utilities (costs)),

- to test more realistic models of public service systems (capacity and time constraints, hierarchical systems, etc.),

- to compare other types of fairness criteria, e.g. MMF,
- to examine the sensitivity to input data (network topology, spatial distribution of customers, etc.).

\section{Acknowledgement}

Authors are grateful for financial support by the Grant agency of the Ministry of Education of the Slovak Republic and the Slovak Academy of Sciences (project VEGA 1/0296/12) and by Slovak research and development agency (project APVV-0760-11). We would also like to thank to "Centre of excellence for systems and services of intelligent transport" (ITMS 26220120028) for built up the infrastructure, which was used.

\section{References}

[1] JANACEK, J., JANOSIKOVA, L., BUZNA, L.: Optimized Design of Large-Scale Social Welfare Supporting Systems on Complex Networks. In: Thai, M.T. Pardalos, P.M. (eds.) Handbook of Optimization on Complex Networks, Springer Media and Business, 2012.

[2] VETSCHERA, R.: A General Branch-and-Bound Algorithm for Fair Division Problems. Computers and Operations Research, 37, pp. 2121-2130, 2010.

[3] NACE, D., DOAN, L.N., KLOPFENSTEIN, BASHLLARI, A.: Max-Min Fairness in Multi-Commodity Flows.

[4] Kelly, F. P., MAULlOO, A. K., TAN, D. K. H.: Rate Control for Communication Networks: Shadow Prices, Proportional Fairness and Stability. J. of the Operational Research Society, 49 (3), pp. 237-252, 1998.

[5] BERTSIMAS, D., FARIAS, V.F., TRICHAKIS, N.: The Price of Fairness. Operations Research, 59 (1), pp. 17-31, 2011.

[6] DASKIN, M., S.: Network and Discrete Location. Models, Algorithms, and Applications. John Wiley \& Sons, New York, NY, 1995.

[7] JANACEK, J., BUZNA, L.: An Acceleration of Erlenkotter-Koerkel's Algorithms for the Uncapacitated Facility Location Problem. Annals of Operations Research, 164 (1), pp. 97-109, 2008.

[8] United states census database 2000. http://www.nationalatlas.gov/mld/ce2000t.html. 